\title{
Acupuncture for Functional Gastrointestinal Disorders
}

\section{Toku TAKAHASHI}

Professor, Department of Surgery, Medical College of Wisconsin, Milwaukee, Wisconsin

\section{Introduction}

Complementary and Alternative Medicine (CAM), which includes Acupuncture, Herb, Shiatsu, Massage, Yoga and some others, is getting more common and common in the United States. Acupuncture is an empirical medical procedure that has been practiced in Japan and China for thousands of years and has been rapidly accepted throughout Western countries, including the United States. In November 1997, NIH held a consensus conference regarding acupuncture and issued a statement that acupuncture is effective for treating nausea and vomiting associated with postoperative and chemotherapy (N.I.O. Health 1997, Morey 1998). Acupuncture has been used for treating functional gastrointestinal (GI) disorders, including irritable bowel syndrome (IBS) and functional dyspepsia (FD) (Diehl 1999, Takahashi 2006). It is conceivable that acupuncture is effective in patients with functional GI disorders because it has been shown to alter acid secretion, GI motility and visceral pain. However, the precise mechanism of acupuncture on GI function remains to be clarified.

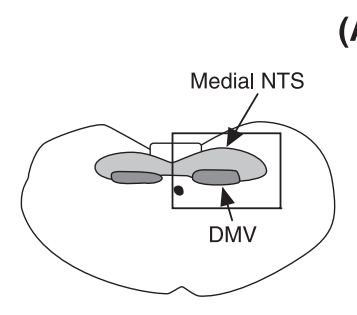

(A)

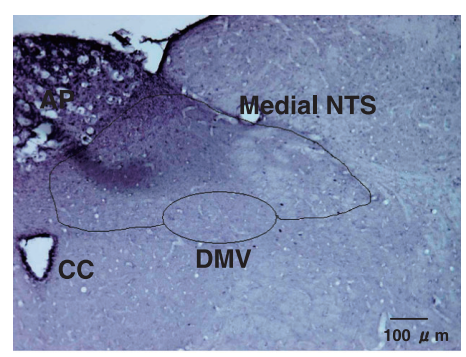

(B)

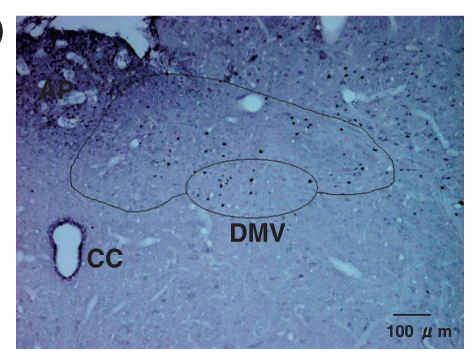

II. Effects of acupuncture on gastric functions of normal rats and dogs

Acupuncture at ST-36 stimulates gastric contractions in conscious rats acupuncture induced-contractions were antagonized by vagotomy and hexamethonium and atropine, but not splanchnic ganglionectomy and - L-NNA and guanethidine.

Acupuncture-evoked afferent input converges in several nuclei in the brain stem, which play an important role in mediating acupuncture-induced GI responses. The nucleus tractus solitarius (NTS) is the primary brainstem relay for visceral information from cardiovascular, respiratory and GI systems. The NTS is adjacent to the dorsal motor nucleus of the vagus (DMV) and composes the dorsal vagal complex (DVC). The DVC integrates vago-vagal reflex, which play a major role in regulation of GI function. The NTS also receives somatic afferent inputs (Gamboa 2001). The NTS neurons are activated by cutaneous mechanical stimulus to the hind limb in rats (Toney 2000). The neurons of the NTS and the spinal cord were labeled by injection of

\section{Control}

Acupuncture at ST-36
Fig. 1. c-Fos immunohistochmistry in the NTS and DMV of control rats (A) and acupuncute-treated rats (B). In rats treated with EA at ST-36, c-Fos immunopositive cells of NTS and DMV were significantly increased, compaired with those of control rats. (Iwa et al; Auton Neurosci 137, 67-76, 2007) 
neuro-anatomical tracers to the hind limb in rats (Lee 2001). These suggest that somatic stimulation induced by acupuncture is conveyed to the NTS through the spinal cord. It has been shown that acupuncture at the hind limbs stimulates GI motility in rats (Sato 1975, Tatewaki 2003). c-Fos immunohistochemistry of the brain stem showed the anatomical evidences of neural pathways in mediating acupuncture-induced gastric motor responses (see Figure 1, Iwa 2007). Acupuncture at the hind limb (ST-36) increased the number of c-Fos immunopositive cells at the DMV and the medio-caudal and caudal NTS.

These results suggested that somatic afferents activated by acupuncture at ST-36 is conveyed to the medio-caudal and the caudal NTS and stimulates the DMV neurons (see Figure 1, Iwa 2007). Thus, acupuncture at ST-36 stimulates gastric motility via the DMV and vagal efferent in normal condition rats.

III. Effects of acupuncture on stress inducedgastric disorders of rats and dogs

Functional GI disorders are multi-factorial in which the pathophysiological mechanisms are variably

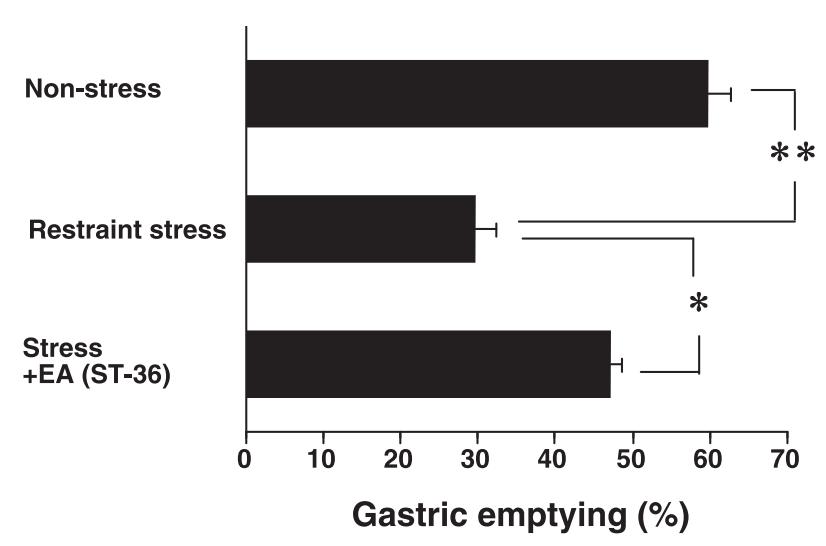

Fig. 2. Effects of EA at ST-36 on delayed gastric emptying induced by restraint stress. Restraint stress significantly delayed gastric emptying. EA at ST-36 improved stressinduced delay of gastric emptying. (Iwa et al; Dig Dis Sci 51, 1493-1500, 2006)

combined in different patients. Approximately 30$50 \%$ of patients with FD have motor disorders, such as delayed gastric emptying and impaired gastric accommodation to a meal (Tack 1998). In addition to the postprandial dysmotility, gastric contractions in the interdigestive period (migrating motor complex; MMC) are also impaired in subset of FD patients (Kusano 1997).
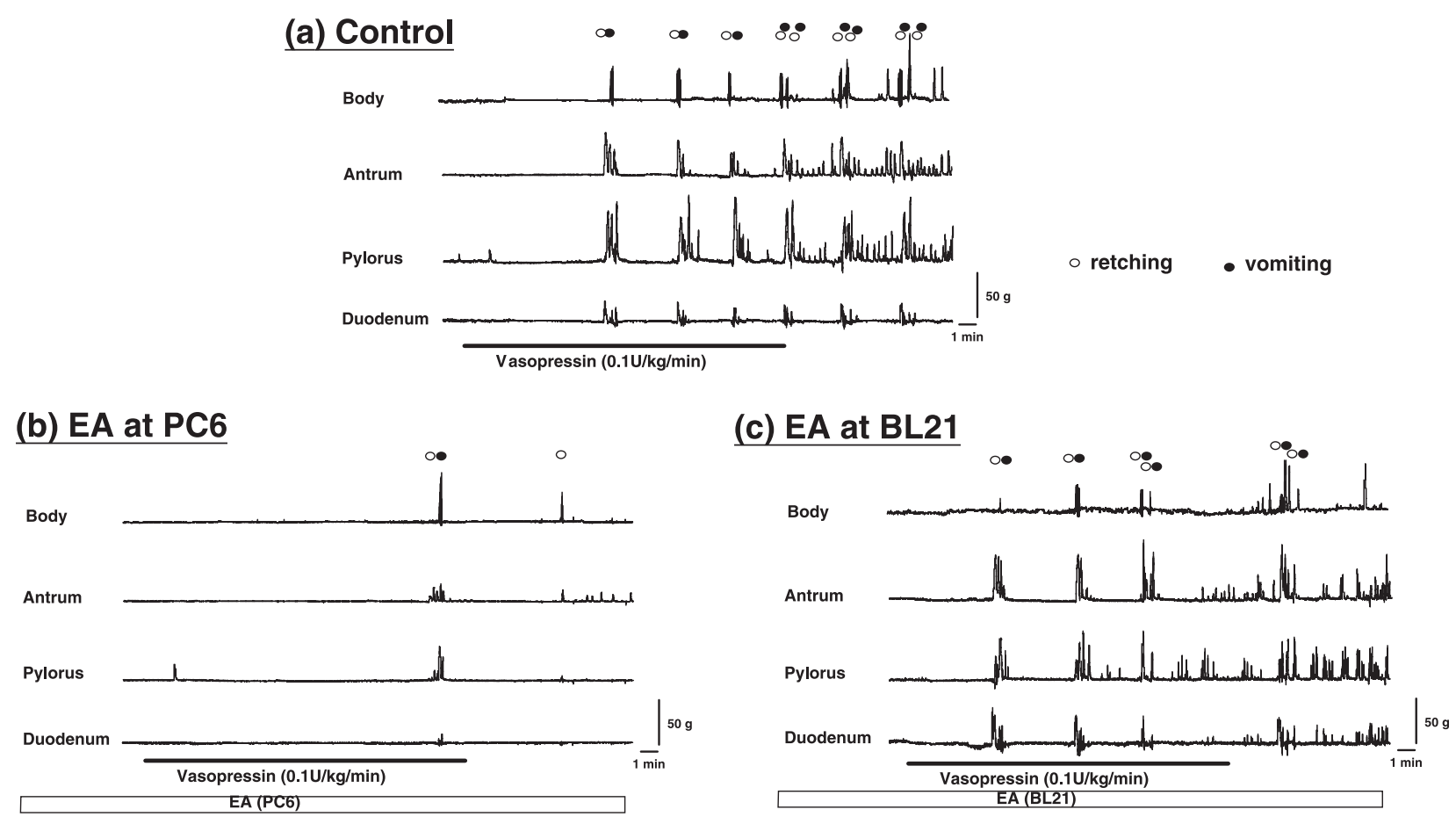

Fig. 3. Effects of electro-acupuncture (EA, $10 \mathrm{~Hz}$ ) at PC-6 on retching and vomiting induced by vasopressin in dogs. Vasopressin infusion evoked retrograde peristaltic contractions resulting in vomiting and retching (a). EA at PC-6 (b), but not at BL-21 (c), significantly attenuated the retrograde peristaltic contractions and the frequency of retching and vomiting induced by vasopressin. (Tatewaki et al; Am J Physiol 288, R401-408, 2005) 
Gastric emptying was delayed by restraint stress in rats (see Figure 2, Iwa 2006). Acoustic stress abolished the occurrence of gastric MMC in conscious dogs - heart rate variability (HRV) analysis showed that parasympathetic nerve activity (HF components) was reduced and sympathetic nerve activity (LF components) was increased during acoustic stress loading in conscious dogs.

Delayed gastric emptying induced by restraint stress was significantly improved by acupuncture at ST-36 in rats Acupuncture also recovered impaired MMC induced by acoustic stress in dogs HF components were increased and LF components were decreased by acupuncture during acoustic stress loading. These results suggest that GI disorder, are developed by stress, and that acupuncture improves GIdisorderby stimulating parasympathetic pathway.

\section{Effects of acupuncture on vasopressin induced-emesis and rectal distension- induced-blood pressure changes}

In conscious dogs, acupuncture at PC-6 prevents emesis induced by vasopressin. The anti-emetic effect of acupuncture is mediated via a central opioid pathway (see Figure 3, Tatewaki 2005, Takahashi 2007). Acupuncture at ST-36 reduces rectal distensioninduced blood pressure changes in conscious dogs. The anti-nociceptive effect of acupuncture is also mediated via a central opioid pathway (see Figure 4, Iwa 2005). (a)

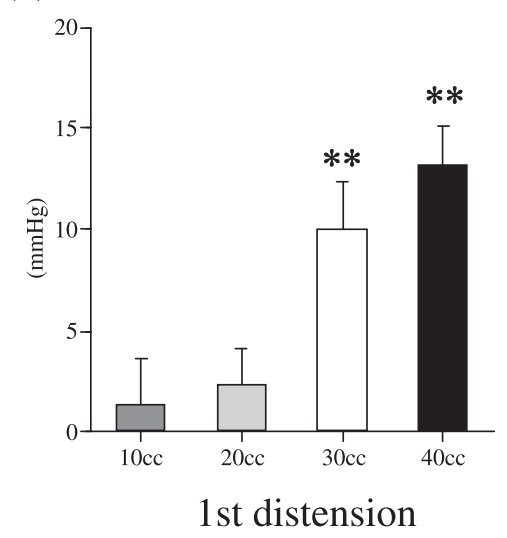

(d)

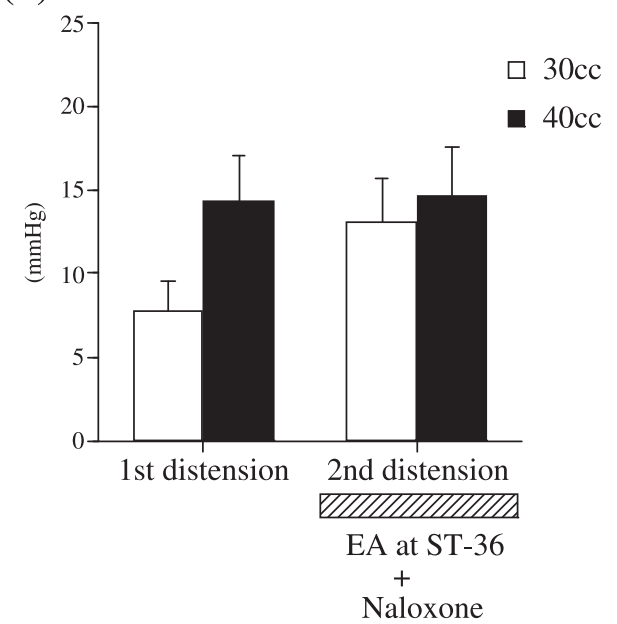

(c)
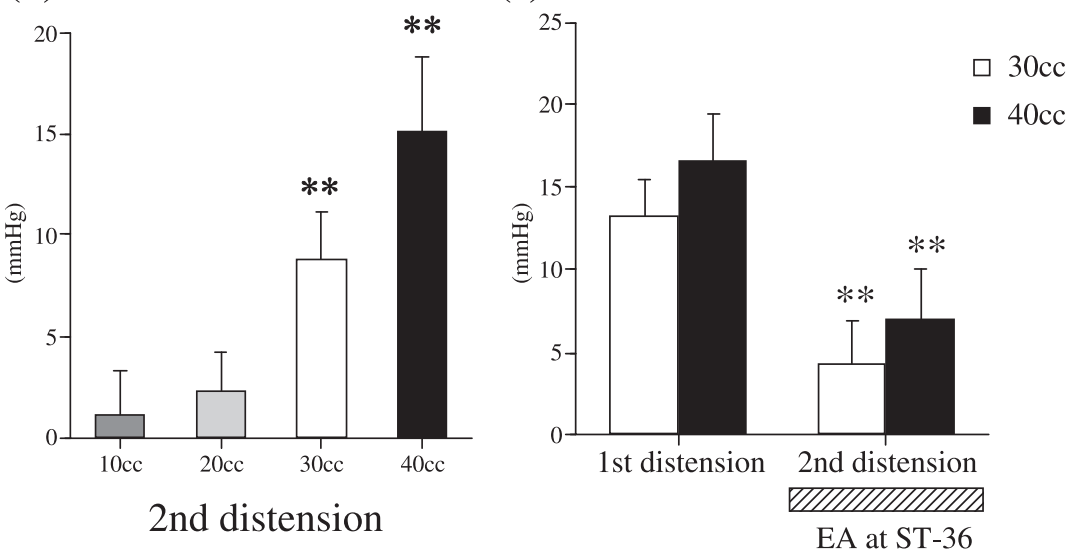

Fig. 4. Effects of EA on rectal distension-induced blood pressure increase in conscious dogs. The increase in blood pressure was similar between the reponse to the first (a) and second (b) rectal distension. Rectal distension induced-increase of blood pressure was significantly reduced by EA at ST-36 (c). By the pretreatment of naloxone, the inhibitory effect of EA at ST-36 was no more observed in response to rectal distension (d). In contrast, the treatement of naloxone methiodide, rectal distension induced-increase of blood pressure was significantly reduced by EA at ST-36 (e). (Iwa et al; Dig Dis Sci 50, 1264-1270, 2005) 
V. Conclusion

In subsets of patients with functional dyspepsia (FD), gastric motility is impaired. The stimulatory effects of acupuncture at ST-36 on GI motility may be beneficial in patients with $\mathrm{FD}$, as well as constipation-predominant IBS patients who show delayed colonic transit. Anti-emetic effects of acupuncture at PC-6 may be beneficial in patients with FD. Anti-nociceptive effects of acupuncture at ST-36 may be beneficial in patients with visceral hypersensitivity. In the future, it is expected that acupuncture will be used in the treatment of patients with functional GI disorders.

\section{References}

N. I. O. Health, Acupuncture, NIH consens. Statement. 15: 1-34, 1997.

Morey, S. S. NIH issues consensus statement on acupuncture. Am Fam Physician. 57: 2545-2546, 1998.

Diehl, D. L. Acupuncture for gastrointestinal and hepatobiliary disorders. J Altern Complement Med. 5: 27-45, 1999.

Takahashi, T. Acupuncture for functional gastrointestinal disorders. J Gastroenterol. 41: 408-417, 2006.

Gamboa-Esteves, F. O., Lima, D., and Batten, T. F. Neurochemistry of superficial spinal neurones projecting to nucleus of the solitary tract that express c-fos on chemical somatic and visceral nociceptive input in the rat. Metab Brain Dis. 16: 151-164, 2001.

Toney, G. M., and Mifflin, S. W. Sensory modalities conveyed in the hindlimb somatic afferent input to nucleus tractus solitarius. J Appl Physiol. 88: 2062-2073, 2000.

Lee, C. H., Jung, H. S., Lee, T. Y., Lee, S. R., Yuk, S. W., Lee, K. G., and Lee, B. H. Studies of the central neural pathways to the stomach and Zusanli (ST36). Am J Chin Med. 29: 211-220, 2001.

Sato, A., Sato, Y., Shimada, F., and Torigata, Y.
Changes in gastric motility produced by nociceptive stimulation of the skin rats. Brain Res. 87: 151-159, 1975.

Tatewaki, M., Harris, M., Uemura, K., Ueno, T., Hoshino, E., Shiotani, A., Pappas, T. N., and Takahashi, T. Dual effects of acupuncture on gastric motility in conscious rats. Am J Physiol Regul Integr Comp Physiol. 285: R862-872, 2003.

Iwa, M., Tateiwa, M., Sakita, M., Fujimiya, M., and Takahashi, T. Anatomical evidence of regional specific effects of acupuncture on gastric motor function in rats. Auton Neurosci. 137: 67-76, 2007.

Tack, J., Piessevaux, H., Coulie, B., Caenepeel, P., and Janssens, J. Role of impaired gastric accommocation to a meal in functional dyspepsia. Gastroenterology. 115: 1346-1352, 1998.

Kusano, M., Sekiguchi, T., Kawamura, O., Kikuchi, K., Miyazaki, M., Tsunoda, T., Horikoshi, T., and Mori, M. Further classification of dysmotilitylike dyspepsia by interdigestive gastroduodenal manometry and plasma motilin level. Am J Gastroenterol. 92: 481-484, 1997.

Iwa, M., Nakade Y., Pappas T.N., and Takahashi, T. Electroacupuncture elicits dual effects: stimulation of delayed gastric emptying and inhibition of accelerated colonic transit induced by restraint stress in rats. Dig Dis Sci. 51: 1493-1500, 2006

Tatewaki, M., Strickland, C., Fukuda, H., Tsuchida, D., Hoshino, E., Pappas, T. N., and Takahashi, T. Effects of acupuncture on vasopressin-induced emesis in conscious dogs. Am J Physiol Regul Integr Comp Physiol. 288: R401-408, 2005.

Takahashi, T., Tsuchida, D., and Pappas, T. N. Central effects of morphine on GI motility in conscious dogs. Brain Res. 1166: 29-34, 2007.

Iwa, M., Strickland, C., Nakade, Y., Pappas, T. N., and Takahashi, T. Electroacupuncture reduces rectal distension-induced blood pressure changes in conscious dogs. Dig Dis Sci. 50: 12641270, 2005. 